\title{
Albumin-bilirubin index and platelet-albumin-bilirubin index contribute to identifying survival benefit candidates in patients with hepatocellular carcinoma and Child-Pugh grade $A$ undergoing transcatheter arterial chemoembolization with sorafenib treatment
}

\author{
Keshu Hu ${ }^{1,2 \#}$, Jia Yuan ${ }^{1,2 \#}$, Bei Tang ${ }^{1,2}$, Feng Zhang ${ }^{1,2}$, Shenxin Lu ${ }^{1,2}$, Rongxin Chen ${ }^{1,2}$, Lan Zhang ${ }^{1,2}$, \\ Zhenggang Ren ${ }^{1,2}$, Xin Yin ${ }^{1,2}$ \\ ${ }^{1}$ Liver Cancer Institute \& Zhongshan Hospital, Fudan University, Shanghai, China; ${ }^{2}$ Key Laboratory of Carcinogenesis and Cancer Invasion, \\ Ministry of Education, Shanghai, China \\ Contributions: (I) Conception and design: K Hu, X Yin; (II) Administrative support: R Chen, Z Ren, X Yin; (III) Provision of study materials or \\ patients: J Yuan, S Lu; (IV) Collection and assembly of data: B Tang, F Zhang; (V) Data analysis and interpretation: K Hu, F Zhang, L Zhang; (VI) \\ Manuscript writing: All authors; (VII) Final approval of the manuscript: All authors. \\ "These authors contributed equally to this work. \\ Correspondence to: Xin Yin. Liver Cancer Institute \& Zhongshan Hospital, Fudan University, 136 Yi Xue Yuan Road, Shanghai 200032, China. \\ Email: yin.xin@zs-hospital.sh.cn.
}

Background: Combined therapy of transcatheter arterial chemoembolization (TACE) with sorafenib is used for a large number of patients with intermediate-stage or advanced-stage hepatocellular carcinoma (HCC), but its implementation is limited by the evaluation of pre-treatment liver function reserve. This study aimed to validate the performance of the albumin-bilirubin index (ALBI) and platelet-albuminbilirubin index (PALBI) in predicting survival benefits in patients with HCC and Child-Pugh grade A receiving TACE combined with sorafenib treatment.

Methods: From 2004 to 2018, 418 patients with intermediate/advanced-stage HCC and Child-Pugh grade A receiving TACE combined with sorafenib treatment were retrospectively enrolled. The predictive performance of ALBI and PALBI was explored by survival analysis and receiver operating characteristic curve (ROC) analysis.

Results: The median overall survival (mOS) was 24 and 12 months in patients with ALBI grade 1 and grade 2, respectively. The mOS of patients with PALBI grade 1, grade 2, and grade 3 was 23, 16, and 7 months, respectively. The multivariate analysis showed that ALBI grade 2 [hazard ratio (HR) $=1.39,95 \%$ confidence interval (CI): 1.11-1.74] and PALBI grade 3 (HR =3.72, 95\% CI: 2.26-6.06) were associated with unfavorable prognosis. The ROC analysis revealed that ALBI and PALBI scores had better prediction performance compared with the Child-Pugh score. Subgroup analysis confirmed that by using ALBI or PALBI, patients could be stratified into subgroups with different liver function reserves and distinctive prognosis, regardless of Barcelona Clinic Liver Cancer (BCLC) stage, combination modality, or $\alpha$-fetoprotein (AFP) levels.

Conclusions: Both ALBI and PALBI could predict prognosis in patients with HCC and Child-Pugh grade A receiving TACE and sorafenib. Patients with ALBI or PALBI grade 1 harbored a more favorable survival outcome compared with those with ALBI or PALBI grade 2-3, and hence should be recommended as the best candidates for TACE combined with sorafenib treatment.

$\wedge$ ORCID: 0000-0003-4576-3688. 
Keywords: Liver function; platelet-albumin-bilirubin index (PALBI); prognosis; sorafenib; transcatheter arterial chemoembolization (TACE)

Submitted Apr 03, 2020. Accepted for publication Nov 13, 2020.

doi: $10.21037 / \mathrm{atm}-20-3118$

View this article at: http://dx.doi.org/10.21037/atm-20-3118

\section{Introduction}

Hepatocellular carcinoma (HCC) is one of the most deadly forms of malignant tumors worldwide, and the prognosis of patients with HCC is generally evaluated by the clinical stage (1). The staging systems, for example, the Barcelona Clinic Liver Cancer (BCLC), usually take the tumor stage, liver function, and physical status into account for assessment (2). Specifically, liver function is a major concern during the treatment process, including tumor staging, therapeutic decision making, post-treatment effectiveness, and incidence of adverse events (AE).

According to the BCLC guideline recommendation, transcatheter arterial chemoembolization (TACE) is the standard therapy for intermediate-stage HCC, and molecular target therapy is the first-line treatment for advanced-stage HCC (3). However, based on the HCC guidelines in most Asian countries (4-7), TACE is recommended as an important locoregional treatment for unresectable HCC. The results from the BRIDGE study (8) revealed that approximately $70 \%$ of patients with HCC received TACE treatment in real-world practice; most of these were diagnosed with intermediate- or advancedstage HCC. Evidence from retrospective and prospective studies $(9,10)$ showed that treatment benefits of TACE combined with sorafenib therapy could be achieved in selected patients with HCC. Mechanically, TACE and sorafenib act synergistically to inhibit HCC proliferation and angiogenesis. TACE causes hypoxia, leading to an upregulation of hypoxia-inducible factor 1, which in turn upregulates vascular endothelial growth factor and platelet-derived growth factor receptor, the two important molecular targets of sorafenib (11). However, the survival data from SPACE (12) and START (13) clinical trials showed that TACE with sorafenib treatment did not yield better outcomes compared with TACE alone. In fact, given the high heterogeneity in liver functional reserve and tumor burden, this combination treatment is not beneficial for all patients with intermediate/advanced-stage HCC. A previous systematic review (14) revealed that the incidence of severe liver dysfunction induced by TACE with sorafenib treatment increased to $15 \%$, which was significantly higher than the $5 \%$ in patients with HCC undergoing TACE monotherapy (4), suggesting that aggressive combination treatment could result in severe liver dysfunction, thus offsetting treatment benefits. The best candidates for TACE combined with sorafenib treatment have not been defined.

The Child-Pugh grade is a widely used classification for assessing liver function reserve (15). However, it was originally designed to assess the prognosis of patients with liver cirrhosis. It was proposed to have three major defects (16): subjective variables (hepatoencephalopathy and ascites), arbitrary cutoff values (albumin, bilirubin, and prothrombin time), and a neglect for the cause of cirrhosis [hepatitis B virus ( $\mathrm{HBV}$ ), hepatitis $\mathrm{C}$ virus, autoimmune hepatitis, etc.]. In 2015, based on an extensive international cohort of patients with HCC, Johnson et al. proposed a new liver function assessment method, the albumin-bilirubin index (ALBI) grade. ALBI grade is calculated based on two objective laboratory tests: serum albumin and bilirubin levels (17). The platelet-albumin-bilirubin index (PALBI) grade was proposed by Roayaie on the basis of the ALBI grade (18). In contrast, the calculation of the ALBI or PALBI grade, though considered complicated, avoids the disadvantages of "the ceiling effect" or "the flooring effect" by the arbitrary cutoffs, which may guarantee an accurate evaluation of liver function (16).

The predictive values of ALBI and PALBI grades have been investigated in patients with HCC undergoing different treatment modalities such as TACE (19), radiotherapy (20), or hepatic resection (21). However, data regarding their use in patients undergoing TACE with sorafenib treatment are scarce. This study was performed to evaluate the prognostic prediction values of ALBI and PALBI grades in patients with HCC and Child-Pugh A receiving TACE with sorafenib treatment, aiming to define the ideal candidates for this combination therapy. This article has been presented in accordance with the STROBE reporting checklist (available at http://dx.doi.org/10.21037/ atm-20-3118). 


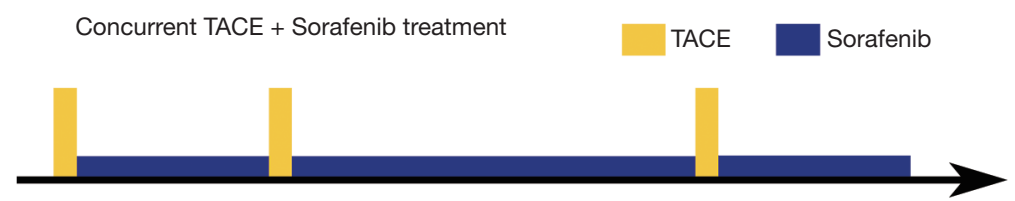

Subsequent TACE + Sorafenib treatment

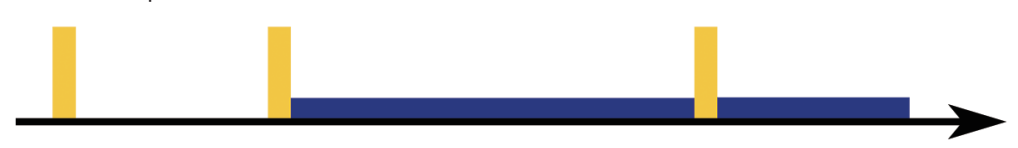

Figure 1 The two combination modalities of sorafenib used for the patients enrolled. TACE, transcatheter arterial chemoembolization.

\section{Methods}

\section{Patients}

From 2004 to 2018, 572 patients with HCC undergoing conventional TACE with sorafenib treatment at the Liver Cancer Institute, Zhongshan Hospital, Fudan University, were retrospectively included in this study. The inclusion criteria were as follows: (I) patients were diagnosed with intermediate- or advanced-stage HCC according to the American Association for the Study of Liver Diseases (AASLD) guidelines (22); (II) patients had Child-Pugh score of A5 to A6 before treatment; (III) patients had an Eastern Cooperative Oncology Group performance status (ECOG PS) score of $0-1$; and (IV) patients received TACE combined with concurrent sorafenib treatment for at least 4 weeks. The exclusion criteria were as follows: (I) patients had another malignancy in addition to HCC; (II) patients had severe complications or concomitant conditions of other organs, including but not limited to kidney dysfunction, central nervous system dysfunction, heart failure, respiratory failure, severe thrombocytopenia, or pancytopenia; (III) patients had no records of laboratory tests to calculate pre-treatment ALBI or PALBI grade; or (IV) they received other anti-tumor treatments prior to TACE with sorafenib. As a result, 418 patients with HCC and Child-Pugh A were enrolled. The study was conducted in accordance with the Declaration of Helsinki (as revised in 2013). The study was approved by the Medical Ethics Committee of Zhongshan Hospital affiliated to Fudan University (no. B2016-086), and individual consent for this retrospective analysis was waived.

\section{Treatment and surveillance}

In the present cohort, the initial treatment modalities were decided by experienced hepatologists with more than 10 years of experience, according to current guideline recommendations $(3,4,6,22)$, individual treatment response, and patients' intention to treat. Conventional TACE was performed according to the institutional protocol described elsewhere (23). Sorafenib was applied to patients either at the initiation of TACE treatment (initial TACE + sorafenib) or subsequent to TACE monotherapy (subsequent TACE + sorafenib) due to disease progression (Figure 1). Sorafenib was administered at a dose of $400 \mathrm{mg}$ twice daily within 3-5 days after each session of TACE treatment and continued during the interval of TACE until the tumor progressed or the patient died. Treatment interruptions and dose reductions (to $400 \mathrm{mg}$ once daily) were permitted for mild or moderate adverse drug reactions such as emaciation, leukopenia, hand-foot syndrome (HFS), and so forth. Sorafenib was stopped when severe AE occurred. After the patient completely recovered, sorafenib was given at a lower dosage. The patients were recommended to receive dynamic computed tomography (CT) or magnetic resonance imaging, blood routine test, serum alpha-fetoprotein (AFP), and liver function examination 4 weeks after TACE and at a 2-month interval thereafter for treatment evaluation and tumor surveillance. Chest CT or bone scintigraphy were performed when clinically necessary. The clinical benefits were evaluated according to the modified Response Evaluation Criteria in Solid Tumor (mRECIST) (24). All data were collected from the patients' records, and the follow-up of their survival was conducted by phone calls to ensure accuracy.

\section{ALBI and PALBI}

The ALBI score was calculated using the following equation: $\mathrm{ALBI}=0.66 \times \log _{10}$ bilirubin $-0.085 \times$ albumin . 
The ALBI grade was classified as grade 1 (ALBI score $\leq-2.60)$, grade $2(-2.60<$ ALBI score $\leq-1.39)$, and grade 3 (ALBI score $>-1.39$ ) (17). The PALBI score was calculated using the following equation: PALBI $=2.02 \times \log _{10}$ bilirubin $-0.37 \times\left(\log _{10} \text { bilirubin }\right)^{2}-0.04 \times$ albumin $-3.48 \times$ $\log _{10}$ platelets $+1.01 \times \log _{10}$ platelets. The PALBI grade was classified as grade 1 (PALBI score $\leq-2.53)$, grade $2(-2.53<$ PALBI score $\leq-2.09$ ), and grade 3 (PALBI score $>-2.09$ ) (18). The parameters were expressed in $\mu \mathrm{mol} / \mathrm{L}$ for bilirubin, $\mathrm{g} / \mathrm{L}$ for albumin, and $1,000 / \mu \mathrm{L}$ for blood platelet count.

\section{Statistical analysis}

The continuous variables were presented as an average \pm standard deviation. Overall survival (OS) was assessed from the date of initiation of treatment until the date of death or the date at which patients were last known to be alive. The OS was analyzed using the Kaplan-Meier method, and the hypothesis tests were conducted using the log-rank test. Multivariable analyses were performed using the Cox proportional hazards model to evaluate the hazard ratio (HR). The prediction efficacy was validated using the area under the receiver operating characteristic curve (AUROC). All the statistical analyses were completed using two-sided tests, and a $\mathrm{P}$ value $<0.05$, or respective corrected value referring to the Bonferroni correction, indicated statistical significance. All the statistical analyses were performed using the software Stata 14.0 for Windows (StataCorp, TX, USA).

\section{Results}

\section{Baseline characteristics}

In this study, 418 of the 572 patients were considered eligible according to the aforementioned criteria. The characteristics of the patients are summarized in Table 1. A large number of the enrolled patients were men $(n=370$, $88.5 \%$ ) and had HBV infection ( $\mathrm{n}=354,84.7 \%)$. Meanwhile, most patients in the present study were diagnosed with BCLC stage C $(n=312,74.6 \%)$ and were serum AFP positive (AFP $>20 \mathrm{ng} / \mathrm{mL}, \mathrm{n}=322,77.0 \%$ ). The patients were divided into two groups according to the initial time of sorafenib treatment: $77(18.4 \%)$ patients formed the initial TACE + sorafenib group, whereas $341(81.6 \%)$ patients formed the subsequent TACE + sorafenib treatment group. The median number of TACE sessions was 4 (range, $1-17)$. Sorafenib was discontinued in 11 patients $(2.6 \%)$ due to the occurrence of severe liver dysfunction $(n=3)$, gastrointestinal hemorrhage $(n=4)$, obstructive jaundice $(n=1)$, severe thrombocytopenia $(n=1)$, severe erythema $(\mathrm{n}=1)$, and persistent fever $(\mathrm{n}=1)$. Seventy-one patients $(17.0 \%)$ had to reduce the dosage due to mild or moderate $\mathrm{AE}$, while the other patients $(\mathrm{n}=336,80.4 \%)$ remained on the original dosage during the combined treatment. HFS occurred in $22.7 \%$ ( $n=95)$ of the patients, including grade 1 $(n=35)$, grade $2(n=34)$, grade $3(n=24)$, and grade $4(n=2)$.

\section{OS}

The median OS (mOS) of the entire group was 19 [95\% confidence interval (CI): 16-22] months. The 0.5-, 1-, and 3 -year survival rates were $80.2 \%, 61.4 \%$, and $30.4 \%$, respectively. In the subgroups, the mOS of patients with BCLC-B was 28 (95\% CI: 22-43) months, while that of patients with BCLC stage C was 16 (95\% CI: 12-19) months $(\mathrm{P}<0.05)$. No statistically significant difference was found in the initial TACE + sorafenib and subsequent TACE + sorafenib groups with mOS of 16 (95\% CI: 10-19) months and 20 (95\% CI: 17-23) months, respectively $(\mathrm{P}=0.070)$.

When classified by the ALBI or PALBI grade, the mOS were 24 and 12 months for patients with ALBI grade 1 and ALBI grade 2, respectively $(\mathrm{P}<0.001)$. Similarly, the mOS of patients with PALBI grade 1 , grade 2 , and grade 3 were 23, 16, and 7 months, respectively $(\mathrm{P}<0.001$, Table 2$)$. The $0.5-, 1-$, and 3 -year survival rates revealed that patients with ALBI grade 1 or PALBI grade 1 had a more favorable prognosis compared with patients with ALBI grade 2 or PALBI grade 2/3 (Figure 2 and Table 2). In contrast, patients with PALBI grade 3 had the shortest mOS of merely 7 months among all patients.

\section{Univariate and multivariate analyses}

The potential prognostic factors, including age, sex, HBV infection, tumor marker, tumor characteristics, BCLC stage, treatment modalities, treatment-related HFS, and ALBI/ PALBI grade, were examined in the univariate analyses (Table 3). The results showed that age, serum AFP levels, tumor size, tumor numbers, BCLC stage, tumor thrombus, extrahepatic metastasis, HFS, and ALBI/ PALBI grade were statistically significant in the univariate analyses. Moreover, all factors with a $\mathrm{P}$ value $<0.100$, except tumor thrombus and extrahepatic metastasis correlated with the BCLC stage, were taken into the Cox model for multivariate analysis. The multivariate analysis indicated that ALBI 
Table 1 The baseline characteristics of the patients

\begin{tabular}{|c|c|}
\hline Variables & Patients (N=418) \\
\hline Age (years), mean \pm SD [range] & $54.8 \pm 11.8[24-90]$ \\
\hline \multicolumn{2}{|l|}{ Gender, n (\%) } \\
\hline Male & $370(88.5)$ \\
\hline Female & $48(11.5)$ \\
\hline \multicolumn{2}{|l|}{ HBV infection, $n$ (\%) } \\
\hline No & $64(15.3)$ \\
\hline Yes & $354(84.7)$ \\
\hline \multicolumn{2}{|l|}{ Tumor thrombus, n (\%) } \\
\hline No & $227(54.3)$ \\
\hline Yes & $191(45.7)$ \\
\hline \multicolumn{2}{|l|}{ Extrahepatic metastasis, $\mathrm{n}(\%)$} \\
\hline No & $228(54.5)$ \\
\hline Yes & $190(45.5)$ \\
\hline \multicolumn{2}{|l|}{ Lymph node invasion, n (\%) } \\
\hline No & $368(88.0)$ \\
\hline Yes & $50(12.0)$ \\
\hline \multicolumn{2}{|l|}{ BCLC stage, n (\%) } \\
\hline $\mathrm{B}$ & $106(25.4)$ \\
\hline $\mathrm{C}$ & $312(74.6)$ \\
\hline \multicolumn{2}{|l|}{ Serum AFP level, n (\%) } \\
\hline$\leq 200 \mathrm{ng} / \mathrm{mL}$ & $186(45.7)$ \\
\hline$>200 \mathrm{ng} / \mathrm{mL}$ & $221(54.3)$ \\
\hline Serum albumin level $(g / L)$, mean $\pm S D$ & $38.7 \pm 4.5$ \\
\hline $\begin{array}{l}\text { Serum bilirubin level }(\mu \mathrm{mol} / \mathrm{L}) \text {, median } \\
\text { [range] }\end{array}$ & $13.4[4-50.3]$ \\
\hline $\begin{array}{l}\text { Blood platelet count }(1,000 / \mu \mathrm{L}) \text {, median } \\
\text { [range] }\end{array}$ & 117 [23-462] \\
\hline \multicolumn{2}{|l|}{ ALBI grade, $n(\%)$} \\
\hline 1 & $195(46.7)$ \\
\hline 2 & $223(53.3)$ \\
\hline 3 & $0(0)$ \\
\hline \multicolumn{2}{|l|}{ PALBI grade, $\mathrm{n}(\%)$} \\
\hline 1 & $246(58.9)$ \\
\hline 2 & $148(35.4)$ \\
\hline 3 & $24(5.7)$ \\
\hline
\end{tabular}

Table 1 (continued)
Table 1 (continued)

\begin{tabular}{|c|c|}
\hline Variables & Patients $(\mathrm{N}=418)$ \\
\hline Major size of tumor $(\mathrm{cm})$, median [range] & $5.6[0.5-20]$ \\
\hline \multicolumn{2}{|l|}{ Number of tumors, $\mathrm{n}(\%)$} \\
\hline Single & $100(23.9)$ \\
\hline Multiple & $318(76.1)$ \\
\hline Total TACE times, median [range] & $4[1-17]$ \\
\hline Pre-sorafenib & $2[0-14]$ \\
\hline Post-sorafenib & $1[0-11]$ \\
\hline \multicolumn{2}{|l|}{ Combination model, n (\%) } \\
\hline Initial TACE + sorafenib group & $77(18.4)$ \\
\hline Subsequent TACE+ sorafenib group & $341(81.6)$ \\
\hline
\end{tabular}

grade 2 (HR $=1.39,95 \%$ CI: $1.11-1.74)$ and PALBI grade 3 (HR $=3.72$, 95\% CI: 2.26-6.06), along with younger age at onset, advanced-stage tumors, and higher serum AFP levels, were associated with an unfavorable prognosis, while the incidence of HFS induced by sorafenib was related to a favorable prognosis (Table 3).

\section{ROC analyses and calibration function}

ROC analyses were performed to evaluate the prediction efficacy of ALBI and PALBI grade compared with the classic Child-Pugh score (Table 4 and Figure 3). The 0.5-, 1-, and 1.5-year AUROCs of ALBI and PALBI scores or grades were consistently larger than those of ChildPugh score, indicating good prediction performance of the two liver function assessment methods in patients with HCC undergoing TACE with sorafenib treatment. The calibration function of each scoring system was validated using calibration curves (Figure 4), showing satisfactory capacities of ALBI and PALBI grade as well.

\section{Subgroup analyses}

The subgroup analyses were conducted in patients with HCC in the BCLC-B/C stage group, initial/subsequent TACE + sorafenib treatment group, and group with AFP levels greater/lesser than $200 \mathrm{ng} / \mathrm{mL}$ to evaluate the prognostic 
Table 2 The overall survival of the patients

\begin{tabular}{|c|c|c|c|c|c|}
\hline Subgroups & $\begin{array}{l}\text { Median survival time } \\
{[95 \% \mathrm{Cl}] \text { (months) }}\end{array}$ & \multicolumn{3}{|c|}{ Survival rate (\%) } & $P$ value \\
\hline ALBI grade & & & & & $<0.001$ \\
\hline 1 & 24 [21-31] & 88.0 & 75.5 & 33.9 & \\
\hline 2 & $12[10-16]$ & 73.4 & 49.2 & 27.0 & \\
\hline PALBI grade & & & & & $<0.001$ \\
\hline 1 & 23 [19-29] & 84.4 & 69.4 & 34.3 & \\
\hline 2 & 16 [11-20] & 77.6 & 55.9 & 28.4 & \\
\hline 3 & $7[4-8]$ & 52.8 & 5.4 & 0.0 & \\
\hline
\end{tabular}

$\mathrm{CI}$, confidence interval; ALBI, albumin-bilirubin index; PALBI, platelet-albumin-bilirubin index; NA, not applicable.

A

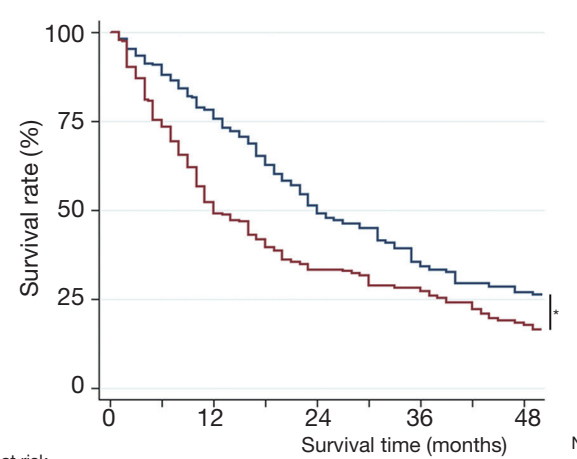

Number at risk

ALBI grade 1

ALBI grade 2
B

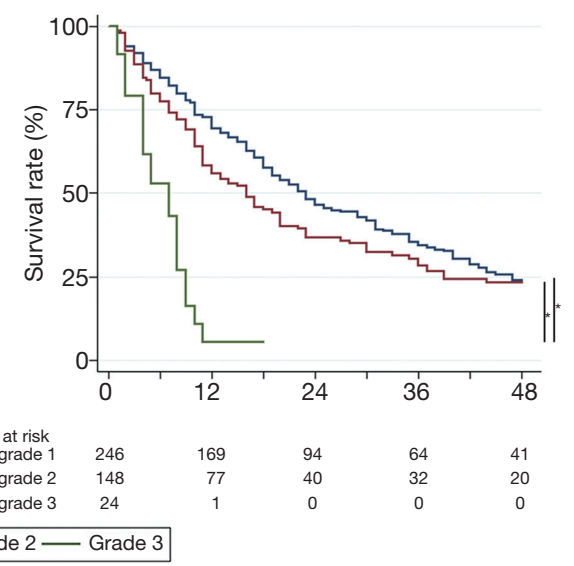

Figure 2 The survival curves by PALBI and ALBI grades. *, statistically significant $(\mathrm{P}<0.05$, or $\mathrm{P}<0.017$ within PALBI grade). PALBI, platelet-albumin-bilirubin index; ALBI, albumin-bilirubin index.

prediction values of ALBI and PALBI grade in HCC. In all subgroups, patients with ALBI grade 1 or PALBI grade 1 had a prolonged OS compared with those with ALBI grade 2 or PALBI grade $2 / 3$, although not all the differences were statistically significant due to the limited patient number in some subgroups (Table 5). The most favorable prognosis was observed in patients with ALBI or PALBI grade 1 and hence these patients should be deemed as the best candidates for the combined treatment of TACE with sorafenib.

\section{Discussion}

With the demand for precision medicine and individualized medicine in the current clinical practice, novel and individualized strategies for nonsurgical therapy of HCC are necessary and urgently needed (25). Referring to the current guidelines $(3,6,26)$, sorafenib is not only recommended for advanced-stage HCC but also applied in patients with intermediate-stage HCC after the TACE refractory status. A recent review suggested that the combined therapy of TACE and sorafenib improved survival in patients with portal vein tumor thrombus (PVTT) (14). Nevertheless, severe liver dysfunction and even treatment-related death occasionally occur after aggressive combination therapy even after a strict selection of candidates based on ECOG score and Child-Pugh grade, thus offsetting treatment benefits and compromising the prognosis of patients with HCC. Hence, the identification of the best candidates for 
Table 3 Univariable and multivariable analyses

\begin{tabular}{|c|c|c|c|c|c|c|}
\hline Prognostic factors & \multicolumn{2}{|c|}{ Univariable } & \multicolumn{4}{|c|}{ Multivariable } \\
\hline Age, years (>50 vs. $\leq 50)$ & $0.72(0.58-0.90)$ & $0.004^{*}$ & $0.64(0.50-0.81)$ & $<0.001^{*}$ & $0.72(0.58-0.92)$ & $0.007^{*}$ \\
\hline Gender (female vs. male) & $1.22(0.89-1.68)$ & 0.217 & NA & - & - & - \\
\hline Number of tumors (multiple vs. single) & $0.68(0.53-0.88)$ & $0.003^{*}$ & $0.76(0.57-1.01)$ & 0.058 & $0.79(0.61-1.03)$ & 0.086 \\
\hline BCLC stage (BCLC-C vs. BCLC-B) & $1.76(1.35-2.28)$ & $<0.001^{*}$ & $1.18(0.86-1.62)$ & 0.296 & $1.41(1.06-1.87)$ & $0.019^{\star}$ \\
\hline Lymph node invasion (yes vs. no) & $0.76(0.50-1.15)$ & 0.191 & NA & - & - & - \\
\hline Hand-foot syndrome (yes vs. no) & $0.40(0.29-0.55)$ & $<0.001^{*}$ & $0.43(0.31-0.61)$ & $<0.001^{*}$ & $0.48(0.35-0.66)$ & $<0.001^{*}$ \\
\hline Serum AFP level, ng/mL (>200 vs. $\leq 200)$ & $2.45(1.94-3.10)$ & $<0.001^{*}$ & $2.16(1.66-2.80)$ & $<0.001^{*}$ & $2.10(1.66-2.68)$ & $<0.001^{\star}$ \\
\hline ALBI grade (2 vs. 1) & $1.50(1.20-1.87)$ & $<0.001^{*}$ & $1.36(1.02-1.81)$ & $0.035^{\star}$ & $1.39(1.11-1.74)^{\dagger}$ & $0.004^{*}$ \\
\hline \multicolumn{7}{|l|}{ PALBI grade } \\
\hline 2 vs. 1 & $1.17(0.93-1.48)$ & 0.187 & $0.98(0.72-1.32)$ & 0.878 & $1.18(0.92-1.51)$ & 0.183 \\
\hline 3 vs. 1 & $4.79(2.98-7.71)$ & $<0.001^{\star}$ & $2.88(1.65-5.04)$ & $<0.001^{\star}$ & $3.72(2.28-6.06)$ & $<0.001^{*}$ \\
\hline
\end{tabular}

${ }^{*}$, statistically significant $(\mathrm{P}<0.05) ;{ }^{\dagger}$, the partial adjusted HR of ALBI grade was calculated separately with PALBI due to their collinearity. $\mathrm{HR}$, hazard ratio; Cl, confidence interval; HBV, hepatitis B virus; BCLC, Barcelona Clinic Liver Cancer stage; TACE, transcatheter arterial chemoembolization; AFP, alpha-fetoprotein; ALBI, albumin-bilirubin index; PALBI, platelet-albumin-bilirubin index; NA, not applicable.

Table 4 The AUROC of ALBI score (grade), PALBI score (grade) and Child-Pugh score

\begin{tabular}{lccc}
\hline & & \multicolumn{1}{c}{ AUROC at $(95 \% \mathrm{Cl})$} \\
\cline { 2 - 4 } Classification criteria & 0.5 year & 1 year & 1.5 years \\
\hline ALBI score & $0.628(0.566-0.690)$ & $0.668(0.615-0.721)$ & $0.634(0.580-0.687)$ \\
ALBI grade & $0.593(0.539-0.648)$ & $0.628(0.581-0.674)$ & $0.585(0.537-0.633)$ \\
PALBI score & $0.627(0.561-0.692)$ & $0.658(0.605-0.711)$ & $0.649(0.596-0.701)$ \\
PALBI grade & $0.588(0.528-0.648)$ & $0.625(0.577-0.672)$ & $0.598(0.552-0.644)$ \\
Child-Pugh score & $0.578(0.524-0.632)$ & $0.611(0.568-0.653)$ & $0.578(0.537-0.619)$ \\
\hline
\end{tabular}

AUROC, area under the receiver operating characteristic curve; $\mathrm{Cl}$, confidence interval; $\mathrm{ALBI}$, albumin-bilirubin index; PALBI, plateletalbumin-bilirubin index.

the combined therapy of TACE and sorafenib is urgently needed.

In the present study, patients who were considered with well-preserved liver function (Child-Pugh grade
A) still could be further stratified into subgroups with different liver function reserves and distinctive prognosis by the ALBI or PALBI grade. The ROC curve analysis and subgroups analyses consistently demonstrated the 

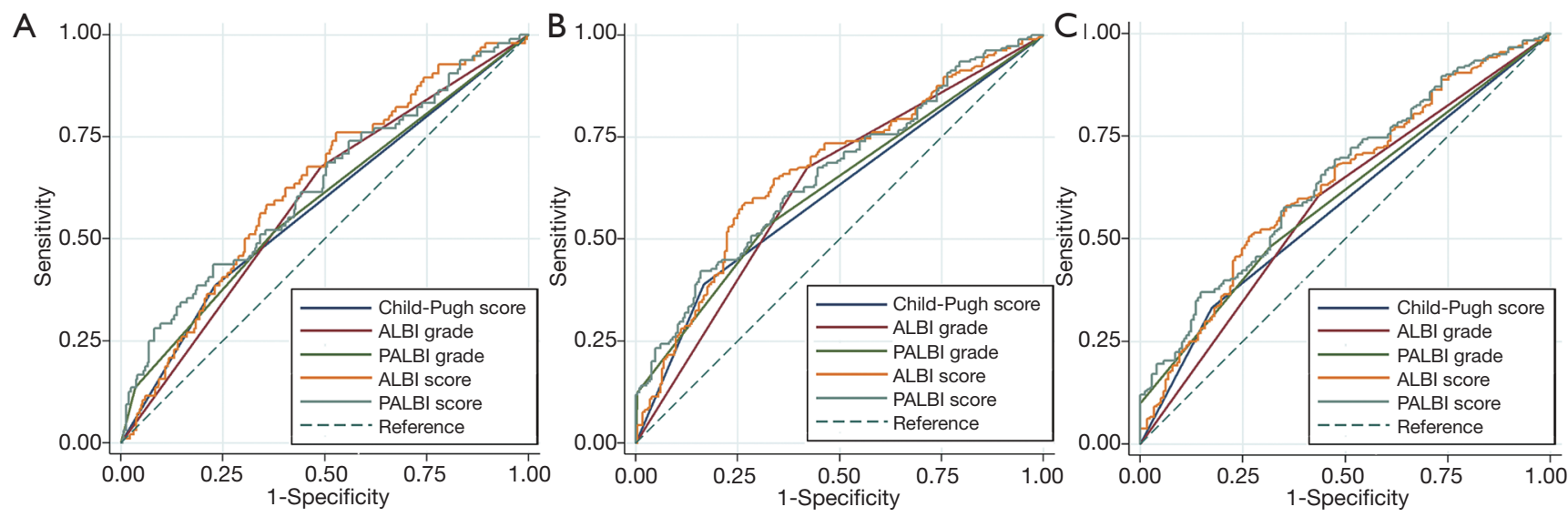

Figure 3 The ROC of Child-Pugh score, ALBI grade, PALBI grade, ALBI score, and PALBI score after 0.5 (A), 1 (B), and 1.5 (C) years. ALBI, Albumin-bilirubin index; PALBI, platelet-albumin-bilirubin index; ROC, receiver operating characteristic curve.
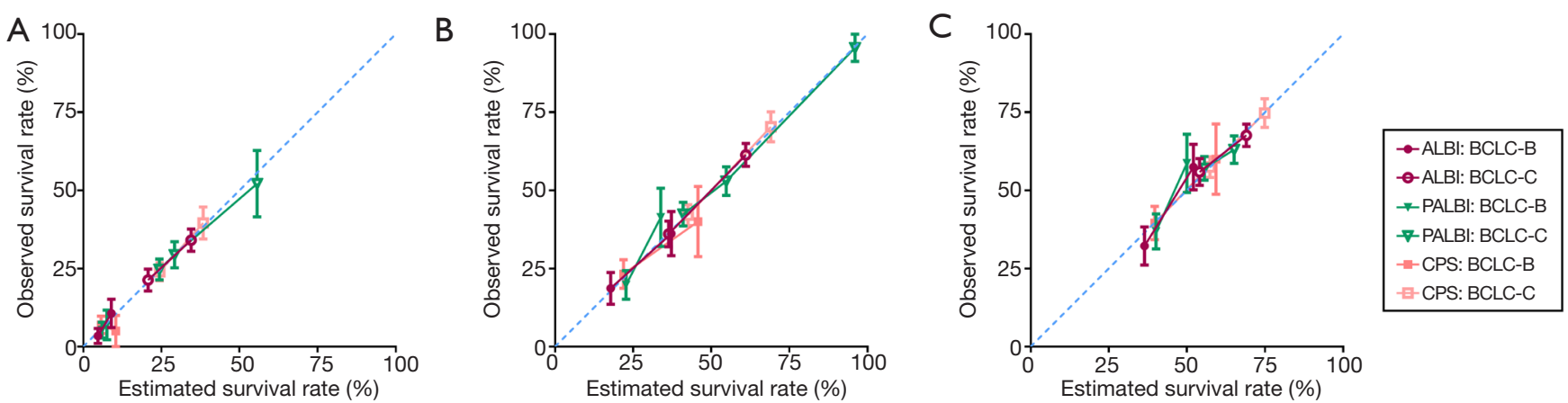

Figure 4 The calibration curves of ALBI grade, PALBI grade, and Child-Pugh score after 0.5 (A), 1 (B), and 1.5 (C) years. The survival could not be estimated for PALBI grade 3 with BCLC-B due to patient number $(n=1)$ nor for PALBI grade 3 with BCLC-C after 1.5 years due to survival (overall survival range, 1-18 months). ALBI, albumin-bilirubin index; BCLC, Barcelona Clinic Liver Cancer stage; CPS, Child-Pugh score; PALBI, platelet-albumin-bilirubin index.

prognostic prediction capacities of ALBI and PALBI grades, highlighting the significant values of the two liver function assessment methods in identifying suitable candidates for TACE with sorafenib treatment.

Currently, an increasing number of patients with intermediate- or advanced-stage HCC are recommended to receive TACE with molecular targeting treatment to improve prognosis, especially in the Asian area, due to the unsatisfactory outcomes of TACE or sorafenib monotherapy. According to the published reports, the mOS for patients undergoing TACE alone was 19.4 months (27) and 7.8 months (10) in BCLC-B and BCLC-C stages, respectively. The $\mathrm{mOS}$ for patients treated with sorafenib alone was 15.3 and 8.6 months in patients with BCLC-B and BCLC-C, respectively (28). Compared with the present series, patients with ALBI grade 1 or PALBI grade 1 achieved a more favorable prognosis when undergoing TACE with sorafenib treatment (ALBI grade 1, mOS 24 months; PALBI grade 1, mOS 23 months, Table 2). Unfortunately, no survival benefits were obtained in patients with PALBI grade 3 or ALBI grade 2. It was proposed that patients with ALBI or PALBI grade 1 were the best candidates for TACE combined with sorafenib treatment in intermediate/advanced-stage HCC.

Compared with the Child-Pugh score, both ALBI and PALBI scores or grades displayed better performance in prognostic prediction for patients with HCC undergoing TACE with sorafenib treatment, as indicated by the ROC analyses. This result could be ascribed to the inherent defects in the Child-Pugh system (29) and the objective 
Table 5 The median overall survival by ALBI or PALBI grade in different subgroups

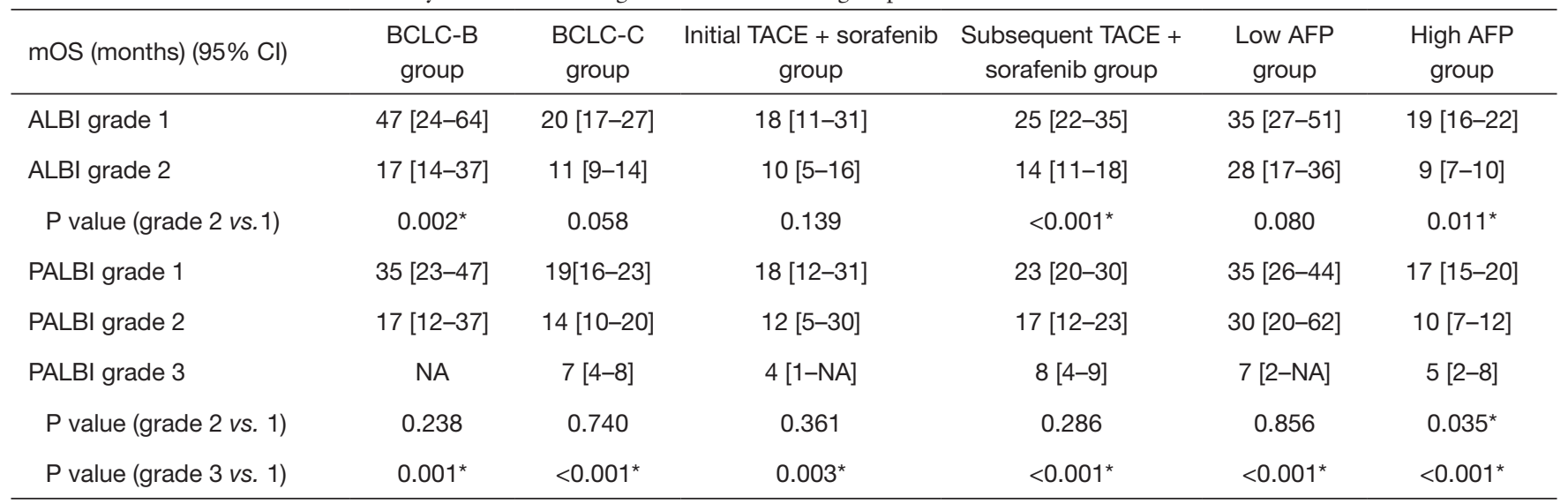

The $P$ values in the table were calculated, referring to the Cox hazard model. *, statistically significant $(P<0.05)$. ALBI, albumin-bilirubin index; PALBI, platelet-albumin-bilirubin index; mOS, median overall survival; Cl, confidence interval; BCLC, Barcelona Clinic Liver Cancer stage; TACE, transcatheter arterial chemoembolization; AFP, alpha-fetoprotein; NA, not applicable.

nature of ALBI or PALBI. Evidence from other studies also revealed that ALBI or PALBI grade possessed better prognostic prediction capacities in patients with HCC undergoing other treatments, such as liver resection, TACE alone, or radiotherapy (19-21). Collectively, it was proposed that ALBI and PALBI grades should be employed in patients with HCC and Child-Pugh grade A for accurate liver function assessment and prognostic prediction.

This study had some limitations. Despite a large sample size, the retrospective nature of this study limited the interpretation and generalization of the results. It only included patients who received TACE combined with sorafenib therapy. The performance of ALBI and PALBI in patients receiving other combination treatments, such as TACE plus lenvatinib or immune checkpoint inhibitor, was not validated. Moreover, the findings were applicable only to conventional TACE and should not be extrapolated to drug-eluting bead TACE or radioembolization procedures. Future prospective studies providing consolidated evidence are necessary to assess the prediction values of ALBI and PALBI grades.

In conclusion, the study investigated the performance of ALBI and PALBI in evaluating liver function reserve and estimating treatment-related survival benefits in patients with Child-Pugh A receiving TACE with sorafenib treatment. It indicated that patients with ALBI or PALBI grade 1 were better candidates for TACE combined with sorafenib treatment compared with those with ALBI grade 2 or PALBI grade 2 or 3 . Considering that no survival benefits were achieved in patients with HCC and ALBI grade 2 or PALBI grade 3, systemic therapy might be a better choice.

\section{Acknowledgments}

The manuscript was edited for proper English language, grammar, punctuation, spelling, and overall style by one or more of the highly qualified native English-speaking editors at MedSci (http://www.medsciediting.com).

Funding: This study was supported by the Natural Science Foundation of China (grant numbers 81672331 and 81972889) and the Excellent Backbone Foundation of Zhongshan hospital, Fudan University (grant number 2019ZSGG20).

\section{Footnote}

Reporting Checklist: The authors have completed the STROBE reporting checklist. Available at http://dx.doi. org/10.21037/atm-20-3118

Data Sharing Statement: Available at http://dx.doi. org/10.21037/atm-20-3118

Peer Review File: Available at http://dx.doi.org/10.21037/atm20-3118

Conflicts of Interest: All authors have filled and submitted the ICMJE uniform disclosure form (available at http://dx.doi. org/10.21037/atm-20-3118). The authors have no conflicts 


\section{Page 10 of 11}

of interest to declare.

Ethical Statement: The authors are accountable for all aspects of the work in ensuring that questions related to the accuracy or integrity of any part of the work are appropriately investigated and resolved. The study was conducted in accordance with the Declaration of Helsinki (as revised in 2013). The study was approved by the Medical Ethics Committee of Zhongshan Hospital affiliated to Fudan University (no. B2016-086), and individual consent for this retrospective analysis was waived.

Open Access Statement: This is an Open Access article distributed in accordance with the Creative Commons Attribution-NonCommercial-NoDerivs 4.0 International License (CC BY-NC-ND 4.0), which permits the noncommercial replication and distribution of the article with the strict proviso that no changes or edits are made and the original work is properly cited (including links to both the formal publication through the relevant DOI and the license). See: https://creativecommons.org/licenses/by-nc-nd/4.0/.

\section{References}

1. Addissie BD, Roberts LR. Classification and staging of hepatocellular carcinoma: an aid to clinical decisionmaking. Clin Liver Dis 2015;19:277-94.

2. Kohla MA, Abu Zeid MI, Al-Warraky M, et al. Predictors of hepatic decompensation after TACE for hepatocellular carcinoma. BMJ Open Gastroenterol 2015;2:e000032.

3. European Association for the Study of the Liver. Electronic address: easloffice@easloffice.eu; European Association for the Study of the Liver. EASL Clinical Practice Guidelines: Management of hepatocellular carcinoma. J Hepatol 2018;69:182-236. Erratum in: J Hepatol. 2019 Apr;70(4):817. doi: 10.1016/j.jhep.2019.01.020. Epub 2019 Feb 7.

4. Omata M, Cheng AL, Kokudo N, et al. Asia-Pacific clinical practice guidelines on the management of hepatocellular carcinoma: a 2017 update. Hepatol Int 2017;11:317-70.

5. Kudo M, Izumi N, Kokudo N, et al. Management of hepatocellular carcinoma in Japan: Consensus-Based Clinical Practice Guidelines proposed by the Japan Society of Hepatology (JSH) 2010 updated version. Dig Dis 2011;29:339-64.

6. Zhou J, Sun HC, Wang Z, et al. Guidelines for Diagnosis and Treatment of Primary Liver Cancer in China (2017 Edition). Liver Cancer 2018;7:235-60.
7. Korean Liver Cancer Study Group (KLCSG); National Cancer Center, Korea (NCC). 2014 KLCSG-NCC Korea Practice Guideline for the Management of Hepatocellular Carcinoma. Gut Liver 2015;9:267-317.

8. Park JW, Chen M, Colombo M, et al. Global patterns of hepatocellular carcinoma management from diagnosis to death: the BRIDGE Study. Liver Int 2015;35:2155-66.

9. Kudo M, Ueshima K, Torimura T, et al. Randomized, open label, multicenter, phase II trial of transcatheter arterial chemoembolization (TACE) therapy in combination with sorafenib as compared with TACE alone in patients with hepatocellular carcinoma: TACTICS trial. J Clin Oncol 2018;36:abstr 4017.

10. Ren B, Wang W, Shen J, et al. Transarterial Chemoembolization (TACE) Combined with Sorafenib versus TACE Alone for Unresectable Hepatocellular Carcinoma: A Propensity Score Matching Study. J Cancer 2019;10:1189-96.

11. Kudo M. Systemic Therapy for Hepatocellular Carcinoma: 2017 Update. Oncology 2017;93 Suppl 1:135-46.

12. Lencioni R, Llovet JM, Han G, et al. Sorafenib or placebo plus TACE with doxorubicin-eluting beads for intermediate stage HCC: The SPACE trial. J Hepatol 2016;64:1090-8.

13. Chao Y, Chung YH, Han G, et al. The combination of transcatheter arterial chemoembolization and sorafenib is well tolerated and effective in Asian patients with hepatocellular carcinoma: final results of the START trial. Int J Cancer 2015;136:1458-67.

14. Zhang X, Wang K, Wang M, et al. Transarterial chemoembolization (TACE) combined with sorafenib versus TACE for hepatocellular carcinoma with portal vein tumor thrombus: a systematic review and meta-analysis. Oncotarget 2017;8:29416-27.

15. Peng Y, Qi X, Guo X. Child-Pugh Versus MELD Score for the Assessment of Prognosis in Liver Cirrhosis: A Systematic Review and Meta-Analysis of Observational Studies. Medicine (Baltimore) 2016;95:e2877.

16. Kim HJ, Lee HW. Important predictor of mortality in patients with end-stage liver disease. Clin Mol Hepatol 2013;19:105-15.

17. Johnson PJ, Berhane S, Kagebayashi C, et al. Assessment of liver function in patients with hepatocellular carcinoma: a new evidence-based approach-the ALBI grade. J Clin Oncol 2015;33:550-8.

18. Carling U, Røsok B, Line PD, et al. ALBI and P-ALBI grade in Child-Pugh A patients treated with drug eluting embolic chemoembolization for hepatocellular carcinoma. 
Acta Radiol 2019;60:702-9. Erratum in: Acta Radiol. 2019 Feb;60(2):NP2. doi: 10.1177/0284185118808210. Epub 2018 Oct 14.

19. Hansmann J, Evers MJ, Bui JT, et al. Albumin-Bilirubin and Platelet-Albumin-Bilirubin Grades Accurately Predict Overall Survival in High-Risk Patients Undergoing Conventional Transarterial Chemoembolization for Hepatocellular Carcinoma. J Vasc Interv Radiol 2017;28:1224-1231.e2.

20. Ho CHM, Chiang CL, Lee FAS, et al. Comparison of platelet-albumin-bilirubin (PALBI), albumin-bilirubin (ALBI), and child-pugh (CP) score for predicting of survival in advanced hcc patients receiving radiotherapy (RT). Oncotarget 2018;9:28818-29.

21. Luo HM, Zhao SZ, Li C, et al. Preoperative plateletalbumin-bilirubin grades predict the prognosis of patients with hepatitis B virus-related hepatocellular carcinoma after liver resection: A retrospective study. Medicine (Baltimore) 2018;97:e226.

22. Heimbach JK, Kulik LM, Finn RS, et al. AASLD guidelines for the treatment of hepatocellular carcinoma. Hepatology 2018;67:358-80.

23. Yin $\mathrm{X}$, Zhang L, Wang YH, et al. Transcatheter arterial chemoembolization combined with radiofrequency

Cite this article as: Hu K, Yuan J, Tang B, Zhang F, Lu S, Chen R, Zhang L, Ren Z, Yin X. Albumin-bilirubin index and platelet-albumin-bilirubin index contribute to identifying survival benefit candidates in patients with hepatocellular carcinoma and Child-Pugh grade A undergoing transcatheter arterial chemoembolization with sorafenib treatment. Ann Transl Med 2021;9(3):237. doi: 10.21037/atm-20-3118 ablation delays tumor progression and prolongs overall survival in patients with intermediate (BCLC B) hepatocellular carcinoma. BMC Cancer 2014;14:849.

24. Lencioni R, Llovet JM. Modified RECIST (mRECIST) assessment for hepatocellular carcinoma. Semin Liver Dis 2010;30:52-60.

25. Eggert T, Greten TF. Current Standard and Future Perspectives in Non-Surgical Therapy for Hepatocellular Carcinoma. Digestion 2017;96:1-4.

26. Vogel A, Cervantes A, Chau I, et al. Hepatocellular carcinoma: ESMO Clinical Practice Guidelines for diagnosis, treatment and follow-up. Ann Oncol 2018;29:iv238-55.

27. Lencioni R, de Baere T, Soulen MC, et al. Lipiodol transarterial chemoembolization for hepatocellular carcinoma: A systematic review of efficacy and safety data. Hepatology 2016;64:106-16.

28. Bruix J, Cheng AL, Meinhardt G, et al. Prognostic factors and predictors of sorafenib benefit in patients with hepatocellular carcinoma: Analysis of two phase III studies. J Hepatol 2017;67:999-1008.

29. Kok B, Abraldes JG. Child-Pugh Classification: Time to Abandon? Semin Liver Dis 2019;39:96-103. 